\title{
Introduction to Data Ethics of Power
}

'Imagine a piece of music which expresses love. It is not love for any particular
person. Another piece of music will express another love. Here we have two distinct
emotional atmospheres, two different fragrances, and in both cases the quality of love
will depend upon its essence and not upon its object. Nevertheless, it is hard to con-
ceive a love which is, so to speak, at work, and yet applies to nothing.'

Henri Bergson, 1932

Why do we need to talk about data ethics? The relation between humans and their data and information has always involved social and ethical dilemmas, and it is no novelty that data systems and registers have persistently throughout history reinforced power dynamics in society and also created new ones. Nevertheless, at the beginning of the 21 st century the very shape of our contemporary data systems, their ethical implications and power complexes have rapidly transformed with the advancement of new digital technologies and data, which is why I, in this book, propose that we also need a differently shaped data ethics. A recent and new development is the transformation of all things into data as an effortless, costless and seamless extra layer of life and society. Data at the time of writing no longer just captures politics, the economy, culture and lives - data is their extension. It is ingrained in society in multiple forms in increasingly complex digital systems which have been developed to contain and make sense of large amounts of data and to act on that knowledge. These digital data systems form a key component of decision-making in politics, culture and industries, and also on life trajectories, and consequently, they are also the centre of power negotiations between different interests. As such, this new shape of power should be at the core of any ethical concern with data systems - and at the heart of data ethics.

With this book, I want to find a common ground for debates on the development and status of big data and AI sociotechnical environments by spelling out a 'human approach', which I refer to as a 'data ethics of power'. A data ethics of power is concerned with making visible the power relations embedded in big data and AI sociotechnical infrastructures in order to point to design, business, policy, social and cultural processes that support a human-centric distribution of power. But what does this actually mean? Imagine an AI robot that sieves through pictures containing predominantly white faces deciding what human 'beauty' means. Ponder on an online search system that learns from news articles to recognise words such as 'nanny' and 'receptionist' as 
female and words such as 'architect' and 'financier' as male. Consider an AI assessment model that scores an otherwise brilliant teacher badly because it cannot interpret the social and human dimension of the teacher's work. Reflect on a mass surveillance global intelligence network or a mass political profiling campaign enabled invisibly by social networking sites with the personal data of millions of people. These sociotechnical data systems and practices are ethically questionable. Most often they are 'unfair', certainly they are not morally 'good' but 'bad', and in some contexts they might even be deemed illegal. However, in this book I propose that data ethics is not a legal assessment, neither is it a moral evaluation of the good or the bad. As said, a data ethics of power wants to expose the power relations embedded in big data and AI sociotechnical infrastructures. It aspires to find ways to build things, act and govern in a holistic manner that benefits human societies, or what, with reference to the French philosopher Henri Bergson, I seek to advocate as open love. Love in the most literal and figurative sense of the word. Love for humanity. Love for the planet and its inhabitants. Love for each other. Love for the universe and beyond. Love without a specific interest. Just love. Nothing more and nothing less.

An encouraging turn in the early 21 st century is that the conversation on data ethics has matured into a main topic of public debate and a crucial policy agenda item in Europe and beyond. Remarkably, as I will illustrate throughout the book, an ethical stance on data and data innovation, particularly in the late 2010s, transformed into what was considered Europe's competitive position on a global geopolitical stage. A range of societal stakeholders from industry, academia and civil society to governments and intergovernmental organisations were here presenting and trying to solve the various critical problems in our current data infrastructures: data bias and a lack of multicultural representation, the disempowerment of consumers, democracy challenged by black box algorithms, flawed IT security and data protection, data monopolisation, voter manipulation and many more. Furthermore, the lost opportunities of locked-in data for science, public service, business and, in particular, the technical development of AI systems were presented as major problems in the state of affairs.

The problems were then, and are always in certain critical moments, evident. But how do we then change the direction of a sociotechnical development that we have identified to move contrary to our human values, challenging human dignity, cohesion, agency, responsibility and democracy? Different stakeholders identify different problems and equally diverse solutions are suggested. And in this moment interests are of course bound to clash, because not all problems with our data reality converge and neither do their solutions. This is the moment when real controversy arises, when power takes material form. I propose that to achieve change in the age of big data and AI, we need to take an extra look at this moment, because it is in this controversial moment - when 
critical problems become visible, when value and interest negotiation take centre stage and compromises are made - that actual sociotechnical transformations emerge.

At times it seems too overwhelming - the complexity of issues, challenges and the compromises we have to make. Though, as I will argue in the book, not all is lost to the state of affairs. The book is built on the idea that humans are in control of these compromises - but only if we see the entire field of powers and interests invested in a sociotechnical transformation, including our own. The greatest challenge we have today is ourselves. What I see in the 'governance' efforts of policy and decision-makers in state, intergovernmental, technical, industry and civil society communities is that when attempting to orchestrate the symphony of sociotechnical acts and agents that comprise sociotechnical change, we repeatedly fail to address its complexity. Our vision is clouded by our particular problems and brilliant solutions, and therefore, we do not see the core problem of sociotechnical change, which is the very shape of the power dynamics that effect change and outline the direction of sociotechnical development. We do it over and over again. We undervalue the complexity of the powers embedded in our sociotechnical environments and the multifarious ethical and social implications. We neglect to look beyond our own interest, fields of application and expertise, and because of this we create implications for ourselves, and even produce new ethical problems. Time after time, we overvalue our own - although predominantly well-meaning, but also often very limited - perspectives on problems and solutions. We fail to coordinate, to translate amongst each other and, most crucially, to assess broader social and ethical implications. I will therefore in this book present a point of reference, where we can meet, by spelling out the human approach - a data ethics of power.

The book contributes to debates on data ethics, big data and Artificial Intelligence in three ways:

\section{A Data Ethics of Power}

'Data ethics' at first gained traction in public discourse as a term to address the general socially and ethically problematic sides of big data technologies and systems in addition to their challenges to privacy. Then data ethics came to represent the 'good intention' of primarily companies and states and it became part of an 'ethics by design' applied ethics, a moral philosophy, with methodologies and practices designed to instil good human values into big data and AI systems. In the book, I part from a concern with the morally 'good' and 'bad' of big data and AI and I position data ethics as a concern with the cultural and social power dynamics of big data and AI. Technologies are cultural products and technological practice is embedded in socially ordering cultural systems 
of meaning-making that are lived and experienced by individuals. This is why cultural systems of sociotechnical change and practice per se are relevant as ethical problems that we should seek to solve with an applied data ethics of power. I argue that we need to be ethically concerned with the constitution of big data and $\mathrm{AI}$ infrastructures as cultural systems of a type of social ordering, in which interests of dominant actors in society have the primary advantage while other minority interests are further disadvantaged. Sociotechnical digital data systems are spatial architectures that reinforce and distribute power. They have data cultures that sustain power for some while repressing the freedom and agency of others, and they are the locus of different powerful interests: corporate, governmental and even scientific.

\section{Data Ethical Governance}

Being actively involved in the evolving public debates and policy debates on the ethical implications of emerging data technologies, I also seek to provide an explanation as to why data ethics became the centre of these discussions. By asking and answering this question, I at the same time outline a governance role and function for data ethics in the context of sociotechnical change. As such, I also propose a common ground for the debates and negotiations that I am involved in based on a human morality and approach to the power structures of big data and AI sociotechnical systems.

I want to illustrate what data ethics can do for human governance in the context of sociotechnical change. I examine the public debates and policy agendas of the late 2010s on the infrastructures of the Big Data Society as one 'in-between' phase of the general phases of the sociotechnical development of a large technological system (Hughes, 1983, 1987) in which different technological cultures and approaches compete to gain technological momentum. I argue that human governance of big data and AI adoption and developments entail a critical awareness of the ethical compromises we make in these moment of controversy, as they constitute the cultural compromises invested in the big data and AI sociotechnical infrastructures' technological momentum for consolidation in society. Accordingly, the vigilance of the cultural powers invested in this is vital. I here urge that we enrich a culture of human power in the governance, design and adoption of big data and AI sociotechnical infrastructures.

\section{The Human Approach}

The 'human-centric approach' is a term and a theme that has emerged, particularly in global policy discourses on AI, with no common conceptualisation other than an emphasis on the special role and status of humans. We could, and 
it has been done, argue against such a human-centric approach and its assumed anthropocentricism if it was primarily concerned with the individual human being and the human species as such. However, in the book I propose that we look at the concept in an entirely different way and I provide a supplementary conceptualisation of the human-centric approach, what I call a 'human approach'. The human approach, I suggest, is one concerned with the role of the human as an ethical being with a corresponding ethical responsibility; or in other words, the human approach is not about prioritising the individual human being (it is not about 'individualisation', as Zygmunt Bauman (2000) would have called it) - it is about the human as an ethical being, our human ethical responsibility for not only ourselves but for life and being in general, and it is about prioritising the human dynamic qualities, a human infrastructure of empowerment, in very concrete ways in big data and AI sociotechnical infrastructures. That is, the human approach also encourages, in practical terms, the empowerment of dynamic human moments in their very data design, use and implementation, which does indeed also include, but is not limited to, the empowerment of the individual human being.

A human approach is best expressed in what I refer to as data ethics 'spaces of negotiation' and 'critical cultural moments'. The spaces of negotiation enable critique and negotiation, but they are only possible when 'systems' (material, immaterial, technological, cultural etc.) clash and controversy arises. The critical cultural moments have special human characteristics and are possible when human memory and intuition are privileged and provided time and space to tinker. In practical terms this is expressed in a prioritisation of the human interest in the data of big data and AI infrastructures via the meaningful involvement of human actors in their very data design, use, governance and implementation.

Thus, the approach of a data ethics of power is not just about humans -it is human, which essentially means that it cannot be put aside; neither can it just be applied when considered useful. We need to think of data ethics as a human morality rather than just a social morality (Bergson, 1932/1977). We can formulate data ethics guidelines, principles and strategies, and we can even program artificial agents to act according to moral rules. However, to truly ensure a human-centric distribution of power, data ethics must become more than just a moral obligation, a set of programmed rules. It must be human.

The philosopher Henri Bergson, who I will refer to extensively in the last part of the book, provided in his philosophical writings an excellent illustration of what this means. It does not mean that humans are bestowed with divine gifts. It does not mean that we are non-natural extra earthly beings. All it means is that humans do not only have the same intellect as machines. We have an additional philosophical capacity which is an intuitive one (Bergson, 1896/1991; Deleuze, 1966/1991), the capacity to 'think movement' (Bergson, 
1907/2001, p. 318) by setting into motion our memory (Bergson, 1896/1991). Most importantly, he raised an essential critique of utilitarian approaches to the living, and by doing so, I would argue that he simultaneously provided us with a conceptual map to also understand the limits of the intellectual capacities of both humans and AI, but that only AI cannot exceed.

Finally, what can we achieve with this human approach? With a human approach we counter the closed and exclusive properties of big data and AI systems that sustain a lived reality of control and order of exclusive societies. These sociotechnical systems materialise interests and enact power asymmetries in society. They only represent a slice of a dynamic moving human reality and multiple cultures, yet they act, and are increasingly also adopted as if they were complete. A human approach counters these exclusive tendencies with love. Love is a concept found in all cultures, from the Greek term, agape, signifying unconditional love for all of humanity to the Buddhist term, maitri, expressive of universal loving-kindness. In this book, I use Henri Bergson's concept of open universal 'love' that has no interest, but is universally directed at the whole of humanity and that therefore enables an open and just society (Bergson, 1932/1977).

\section{OUTLINE OF THE BOOK}

I present three core competing structures of power, each with their shape and style - and each with their 'data cultures'. The book consists of three parts, emphasising the following three different characters of power:

1. On power and big data (Chapters 1 and 2)

2. On power and AI (Chapters 3 and 4)

3. On human power and data ethics (Chapter 5)

In the first two chapters of the book, on power and big data, I address the first kind of power, big data sociotechnical infrastructures (BDSTIs) and their cultures and environments. BDSTIs are constituted physically with fibre cables that run across the globe, enabling data collection and access across geographic territories and jurisdictions, and virtually in spaces of flows around which dominant societal functions are increasingly organised (Castells, 2010). BDSTIs also constitute a redistribution of power facilitated by these new technologically mediated configurations of space and time. To design and shape the infrastructural components of BDSTIs is here an essential form of power, and, for example, surveillance powers of state and industry actors are embedded in BDSTIs as a key property of their architecture and design (Haggerty \& Ericson, 2000; Lyon, 2001, 2010, 2014, 2018; Hayes, 2012; Bauman \& Lyon, 2013; Galic et al., 2017, Clarke, 2018, etc.). 
In Chapters 3 and 4, which constitute the second part of the book, on power and $A I$, I focus on the type of power that is concentrated in the emergence of big data artificial intelligence sociotechnical infrastructures (AISTIs). These are, first and foremost, evolutions of the analytical capabilities of BDSTIs, and constituted as BDSTIs, but with components designed to sense the environment in real time, learning and evolving with autonomous or semiautonomous agency.

The two types of sociotechnical infrastructure described in the first four chapters of the book constitute two forms of power that work in different dimensions of human reality and society. While BDSTIs primarily act in our space by transforming all into immobilised digital data, AISTIs also occupy our time by acting on that data to actively shape the past and present in the image of the future. Accordingly, I propose that a core concern of a data ethics of power should be with AISTIs' and BDSTIs' constitution as cultural systems of a type of social ordering, in which interests of dominant actors in society are spatialised and immobilised and thus more difficult to criticise and renegotiate.

Finally, in the last chapter and part of the book I discuss human power and empowerment, which I claim is at the core of a data ethics of power. The human approach of a data ethics of power concerns the role of the human as an ethical being with a corresponding ethical responsibility. However, ethical agency requires special spatial and temporal conditions to flourish. Human power is, in the face of sociotechnical changes, in constant negotiation with the power of BDSTIs and AISTIs. This therefore requires an applied data ethics that ensures the involvement of human actors in the very data design, governance, use and implementation of sociotechnical data systems.

\section{HOW TO ATTACK A COMPLEX DATA ETHICS PROBLEM}

When I started writing this book, I had already been working in the policy and practice field for many years. Data ethics had become a layman's term and critical showdowns with powerful tech giants were finally acceptable in the public debate. Still, even when ethical reflection and social awareness were present, I saw how we often failed to assess across our different cultures of interests the implications of what we did. As described before, we are immersed socially and technologically in sociotechnical and cultural structures of power that limit us in what we do and what we think we can do with technology.

Normally, when scientists approach a problem, the most traditional way to do this is to look at the problem from one specific area of expertise (such as ethnography, law, philosophy, sociology or engineering). What I have realised is that these separate fields are actually also characterising the way people are talking about AI, big data and data ethics in public discourse and in policy in 
general. I have, over a very long period, been exposed to the different languages and traditions of the stakeholders and scientific communities that shape this field, which has educated me to see the idiosyncrasies and language of each group and tradition: how they deferred to one another, but also how they touched upon each other - that is, what they had in common.

What I have tried to do in the book is to integrate different fields into a more organic way to approach a problem. I wanted to create a synthesis of these languages, integrating different traditions, to represent the common voices I have been interacting with. And it has of course been an incredibly complex labour of relating and reporting on the different traditions and synthesising them in a meaningful way. What I have basically done is to try to embrace the complexity of sociotechnical change, which is in no way an easy task.

How do we create a meta-language where, specifically in technology development, you have fundamentally different, sometimes even contradicting, traditions that have to talk about the same problem?

Here, I was particularly inspired by Thomas J. Misa (1988, 1992, 2009), who addresses this complexity with an approach that moves between different levels of analysis. He suggests a 'multi-level' analysis that encompasses both micro and macro perspectives, which he does to overcome what he considers a false dichotomy between two different framings: the relation between humans and machines produced by analyses that adhere strictly to either a micro or macro perspective on technology (Misa, 1988, 2009). Focusing on either the micro-dynamics of, for example, designers and engineers of a technology or, on the other hand, only on larger macroeconomic or ideological patterns, will present very different and often conflicting views on sociotechnical change, he argues. That is, while the former will often not see the wider social conditions and power dynamics for change, the latter will just as often reduce individual nuances and factors by making sense of them in terms of larger societal dynamics only. A multi-scalar analysis, conversely, acknowledges both perspectives.

Three scales of times (micro, meso and macro) are also central to my delineation of a data ethics of power:

On the micro scale, the very design of a technology can be discerned as closed or open to human controversy and negotiation. For example, an AI agent's algorithms and data processing can be a black box and evolve autonomously without human intervention. Alternatively, it could have a human in the loop design, transparency of design, auditability, and personal data control. Focusing on the design of the technology, I focus on the micro time and space in which it is designed and programmed by human designers or when it is implemented by human users. The micro-scale temporal analysis of a data technology here considers whether the very data design and the design process for this are open for cultural value negotiation. For example: Is the design 
process locked in an unquestioned technological data culture? Or is it open for critical assessment in terms of the invested interests in the process?

On the meso scale, institutions, companies, governments and intergovernmental organisations will be open or closed to negotiation of the values and cultural frameworks for their practices. Closed entities will move along the natural state of affairs and seek only basic compliance with law. Open entities will establish initiatives and practices dedicated to value negotiation and ethical reflection in addition to legal compliance. When patterns in ethical reflection on a specific topic are identified across initiatives and entities, 'data ethical governance' may be introduced.

Lastly, sociotechnical developments and change can be analysed on a macro scale of time. Moments of ethical reflection and negotiation here emerge in between crisis and consolidation of a sociotechnical system in society (Hughes, 1983, 1987; Moor, 1985). These moments are critical as they constitute social negotiation and result in cultural compromises, namely, 'the technological momentum' (Hughes, 1983, 1987) that a sociotechnical system needs to evolve. They are also crucial to phases of innovation and development as they constitute the transformation of the sociotechnical system that emerges from a quest to solve the critical problems of the system. We can here think of 'a battle of the systems' in which an old and a new system exist simultaneously in a relationship of 'dialectical tension' (Hughes, 1983, p. 106-39). Or a moment of conflict and resolution not only among engineers but also in politics and polymaking. In these moments of conflict, critical problems are exposed, different interests are negotiated, and they are finally gathered around solutions to direct the evolution of the system. The new system, or the transformation of the old system, evolves out of the problems identified and solved in this phase. These critical problems of the system are not just resolved as technical problems, for example, with the agreement on technical standards with systems requirements, but are in dialogue with political and historical factors.

\section{THE TERMINOLOGY}

If we want to talk about the same thing, we also need a shared terminology. A key objective of this book is to create a common ground for data ethics debates on $\mathrm{AI}$ and data with a focus on human power. Part of this has been to develop a common discourse for a data ethics of power with some key terms that I introduce here.

\section{Data Ethics}

Data ethics addresses the distribution of power and power relations in the Big Data Society and the conditions of their negotiation and distribution. Applied 
data ethics is concerned with making these power relations visible in order to point to design, business, policy, social and cultural processes that support a human(-centric) distribution of power.

\section{Power}

Power is a well-known, contested and theorised concept with roots in various disciplines and sciences, from Hobbes to Marx, Arendt and Foucault and many more. Here, I only explore the latest peak of contemporary theories on power that specifically address contemporary cultures and digital technology. A data ethics of power is concerned with the distribution of power facilitated within new technologically mediated configurations of space and time (or what I call 'BDSTIs' and 'AISTIs'). It recognises value and interest cultural power struggles and negotiations as a core component of sociotechnical change and governance. This conceptualisation of power and technology stems most profoundly from surveillance and critical data studies that address the state of power in the Big Data Society on the level of the micro-design of systems, business, state and engineering data practices and on the level of macro-societal sociotechnical change. In particular, we need to address power in terms of its 'liquidity' (Bauman, 2000; Bauman \& Haugard, 2008; Lyon, 2010; Bauman \& Lyon, 2013). That is, we should concern ourselves with a type of power that is concentrated and engineered by a few power actors, yet also increasingly self-sustained, re-engineered and evolving in (surveillance) cultures (Lyon, 2018) of use, design, governance and imagination, and therefore difficult - but not impossible - to change. Conceptualisations on culture and power I derive from a tradition within cultural studies that addresses the uneven distribution of power in cultural representation, cultural practices and products. Crucially, in this perspective, cultural power is never stable and can always be challenged and redistributed.

\section{Sociotechnical}

Technology is always part of society, just like society is always part of technology. This also means that one cannot understand one without the other. Technology is not only design and material appearance but also sociotechnical; that is, a complex process constituted by diverse social, political, economic, cultural and technological factors (Hughes, 1987, 1983; Bijker et al., 1987; Misa, 1988, 1992, 2009; Bijker \& Law, 1992; Edwards, 2002; Harvey et al., 2017 etc.) 


\section{(Sociotechnical) Infrastructures}

Infrastructures are the virtual and material sociotechnical organisations of the space of societies. They are engineered and directed, but they also evolve in social, economic, political and historical nonengineered dynamic contexts. Specifically, a sociotechnical infrastructure is a particular type of human-made space which is the material and immaterial, engineered and nonengineered processes that evolve in a space of negotiation and struggle between different societal interests, imaginations and aspirations (Star \& Bowker, 2006; Bowker et al., 2010; Harvey et al., 2017 etc.).

\section{BDSTIs (Big Data Socio-Technical Infrastructures)}

In the first part of the book, I introduce the term BDSTIs to refer to sociotechnical infrastructures constituted by big data technologies. They are the primary infrastructures of the flows (Castells, 2010) of global economies and societies cutting across geographic territories, legal jurisdictions and cultures. In the early 21 st century, BDSTIs were increasingly representing and constituting global societies and environments as the mundane background against which social practice, social networking, identity construction, economy, culture and politics were conducted. They were in part institutionalised, in systems requirements standards for information technology (IT) practices, and in regulatory frameworks for data protection, and they were invested with human imagination about the challenges and opportunities of big data.

\section{AISTIs (Big Data Artificial Intelligence Socio-Technical Infrastructures)}

AISTIs is a term I use to describe an evolution of the analytical capabilities of BDSTIs. AISTIs are constituted as BDSTIs but with components designed to sense the environment in real time, learning and evolving with autonomous or semiautonomous agency. While BDSTIs act in space by transforming all into immobilised digital data, AISTIs also occupy time by acting on that data to actively shape the past and present in the image of the future. In the second part of the book, I focus on the history, ethics and development of AISTIs.

\section{Culture}

There are two sides to culture: (1) It is a system that brings together communities with shared conceptual frameworks and resources, and it is an active system with specific priorities, goals and ways of organising the world that are actively imposed in society. (2) Culture is 'a whole way of life' (Williams, 1958/1993). It does indeed consist of prescribed dominant meanings, but 
importantly culture is also the negotiations of these meanings. That is, culture is not just one. It is multifaceted - institutionalised and formalised, and practiced by dominant groups in society - but it is also subcultural and practiced by, for example, minority groups in society. Thus, culture is never stable; it is from the outset a constructed system of meaning-making and is therefore also always up for contestation and social negotiation.

\section{Data Cultures}

The cultures that frame data science, practice and governance we may call 'data cultures'. They are culturally coded conceptual maps of the engineers, data scientists and designers of data systems; deployers of data systems; legislators of data systems; and users of data systems. They are not always shared, and they may even be in conflict. Data cultures are interrelated with societal power negotiation and struggle. The very practices of data scientists and designers are, for example, framed within specific informal or institutionalised cultural systems of meaning-making. Accordingly, the very practice of developing a data system and design is a cultural practice.

\section{The Human Approach}

The 'human-centric' or 'human-centred' approach was a popular term in late 2010s' policy and advocacy discourses on the ethics of AI and big data, used as a way to recentre the sociotechnical developments in these fields on the human interest. In this book, I further explore and conceptualise this term, but I refer to it as the 'human approach'. I do this to emphasise the role of the human as an ethical being with a corresponding ethical responsibility for not only the human living being but also for life and being in general. In practical terms the human approach is associated with the human interest in the data of AI through the involvement of human actors in the very data design, use and implementation of AI. The human approach of a data ethics of power specifically constitutes a critical reflection on the power of technological progress as well as the big data and AI sociotechnical systems we build and imagine.

\section{Values and (Data) Interests}

Values are 'idealised qualities or conditions in the world that people find good' (Brey, 2010, p. 46). They are represented in power struggles over different cultures and worldviews. Interests are held by different actors and represent social power struggles over, for example, material things such as resources. A data ethics of power is particularly concerned with the interests in data invested in 
data design and governance ('data interests', Hasselbalch, 2021). Values and interests are core components of sociotechnical change.

\section{Moral Agent/Agency, Ethical Agent/Agency}

The concept moral agency is often used interchangeably with ethical agency. However, I want to make a distinction between the two to emphasise the difference between two different capacities. I understand a moral agent as one that can only enforce and act according to moral prescription and determination. For example, 'intelligent' nonhuman agents (AI agents) are moral agents, but they are not ethical beings. This is also why I consider data ethics a human responsibility only.

\section{Human Actor/Agent and Nonhuman Actor/Agent}

I deliberately make a distinction between human and nonhuman agents and actors. However, my intention with this very rough distinction between the human and nonhuman is not grounded in a technological nor cultural determinism; rather, it is a semantic trick aimed at disclosing the limits of the moral agency of AISTIs in particular and, in this connection, the importance of human ethical agency and power to change and govern sociotechnical development. Accordingly, despite my distinction between the two, I do recognise technological artefacts as extensions of human agency and intent as well as their increasing indistinguishability within human environments.

\section{Ethical/Data Ethical Governance}

'Ethical governance' (Rainey \& Goujon, 2011; Winfield and Jirotka, 2018) is a multi-actor, reflexive, open-ended (Harvey et al., 2017; Hoffman et al., 2017) and agile process designed to ensure the 'highest standards of behaviour' (Winfield and Jirotka, 2018). It goes beyond just good and effective governance. I define 'data ethical governance' as a form of ethical governance that specifically addresses the complexity of the Big Data Society with infrastructural practices that create human-centric data cultures. 